\title{
Modelos de diversificación energética en Iberoamérica
}

\author{
Fernando BLANCo SiLVA \\ Responsable de Energía y Sostenibilidad \\ Universidad de Santiago de Compostela \\ fernando.blanco.silva@usc.es \\ Alfonso LÓPEZ DÍAZ \\ Profesor de la Universidad Católica de Ávila \\ alfonso.lopez@ucavila.es \\ Sergio ZuBeLZu MíngueZ \\ Ingeniero Agrónomo de la Ingeniería Anser - España \\ sergio@anseringenieros.es \\ David Rodríguez ARriBAS \\ Ingeniero Técnico Industrial de la empresa Transformaciones y Tratamientos Ambientales S.L. (Aréavalo - Ávila) \\ davidr.arribas@gmail.com
}

Recibido: 17 de febrero del 2012

Enviado a evaluar: 20 de febrero del 2012

Aceptado: 27 de julio del 2012

\section{RESUMEN}

La subida de los precios del petróleo y el accidente nuclear de Fukushima han sacado de nuevo a la palestra un viejo debate acerca del aumento del abastecimiento de energía; en el presente artículo apoyamos que la mejor solución es la diversificación de las fuentes de energía, promoviendo las fuentes alternativas de energía al petróleo y en general las eficiencia energética. A modo comparativo estudiamos la estructura enérgica de ocho países latinoamericanos (Chile, Venezuela, España, Portugal, México, Brasil, Argentina y Colombia) mencionando las características más importantes de cada uno de ellos.

Palabras clave: Consumo de energía, dependencia del petróleo, impacto ambiental, gases de efecto invernadero y producción de electricidad.

\section{Models of energetic diversification in Iberoamerica}

\begin{abstract}
Rising oil prices and the Fukushima nuclear accident have brought back to the fore an old debate about the supply of energy, in this paper we think the best solution is to diversify energy sources, promoting alternative energy sources to oil and overall energy efficiency. We have studied the energetic structure of eight Latin American countries (Chile, Venezuela, Spain, Portugal, Mexico, Brazil, Argentina and Colombia) and we have compared them, mentioning the most important features of each.
\end{abstract}

Keywords: Energy consumption, oil dependence, environmental impact, greenhouse gas and electricity. 


\section{Modèles la diversification énergétique en Ibero-Amérique}

\section{RÉSUMÉ}

La hausse des prix du pétrole et du Fukushima accident nucléaire ont ramené au premier plan un vieux débat sur la fourniture d'énergie, dans cet article Nous pensons que la meilleure solution consiste à diversifier les sources d'énergie, la promotion de sources d'énergie alternatives au pétrole et au rendement énergétique global. Nous avons étudié la structure énergétique de huit pays d'Amérique latine (Chili, Venezuela, Espagne, Portugal, Mexique, Brésil, Argentine et Colombie) et nous les avons comparés, en mentionnant les caractéristiques les plus importantes de chacun.

Mots clé: consommation d'énergie, la dépendance au pétrole, l'impact environnemental, le gaz à effet de serre et de l'électricité.

\section{INTRODUCCIÓN: LA IMPORTANCIA DE LA ENERGÍA EN EL TEJIDO PRODUCTIVO}

La energía desempeña un importante papel en la economía considerándose uno de las parámetros más importantes para valorar el desarrollo económico y social de un país; aunque los escenarios son muy diversos debemos caracterizar a las economías en cuanto a la importancia del petróleo, en algunos casos la abundancia de esta materia prima supone un aliciente mientras que la ausencia de esta fuente supone una debilidad muy importante para la economía de cualquier país [1].

A la hora de estudiar las políticas energéticas de los países iberoamericanos debemos remontarnos a las Crisis del Petróleo (1973 y 1979); en estos años los países productores actuaron en forma de cártel y provocaron subidas espectaculares en el precio del barril y el efecto dominó provocó unas consecuencias desastrosas para todos los países importadores [2]. Hasta la década de los setenta el petróleo no suponía un coste importante en los procesos productivos, pero la primera subida de los precios (desde 1,6 $\$$ hasta $9 \$$ en 1973) supone introducir un coste adicional que distorsiona el sector industrial. Como consecuencia de esta subida de precios sólo los países con altos recursos petrolíferos (Venezuela, México ...) podrían permitirse el consumo preferente del petróleo mientras que los dependientes deben buscar alternativas válidas ya que además de introducir un nuevo coste en el proceso productivo hay un problema estratégico muy grave para los países importadores, porque estarían indefensos ante una nueva subida de precios.

En décadas más recientes el petróleo ha sufrido amplias fluctuaciones en ambos sentidos (no obstante la tendencia general ha sido siempre al alza); los efectos de estas subidas en los precios no han sido tan graves porque en general las políticas energéticas han implantado algunas medidas para blindarse ante nuevas subidas de los precios [3].

\section{LAS POLÍTICAS ENERGÉTICAS A PARTIR DE LOS AÑOS OCHENTA}

Después de la segunda Crisis del Petróleo (1979) los países afectados son conscientes de su debilidad ante nuevas subidas del precio del combustible e implantan políticas energéticas que buscan blindar las economías ante estas fluctuaciones aun- 
que el margen de actuación es limitado porque en muchos casos no existían alternativas tecnológicas (en particular para el transporte); en general existen tres tendencias:

I. Los países con recursos propios mantienen una estructura basada en el petróleo y gas natural, como es el caso de México o Venezuela; no obstante en los años noventa incluso estos países se plantean la diversificación en las fuentes de energía para evitar la dependencia excesiva del petróleo [4], [5]

II. Algunos países apuestan decididamente por las centrales nucleares para la producción eléctrica. En esta línea destacamos Francia y Japón, en el primero la energía nuclear supone un $40 \%$ de la energía primaria consumida cada año y en Japón está en el orden del 30\%.

III. La tendencia general es disminuir la dependencia del crudo fomentando el "mix energético", es decir la diversificación de fuentes como son España, Portugal o Chile. En la década de los ochenta se opta por el gas natural y la energía nuclear; a partir de la década de los noventa la apuesta se centra en el consumo de fuentes autóctonas (en particular las renovables), la eficiencia y el ahorro de energía.

Otro hecho significativo en el contexto estudiado es la firma del Protocolo de Kyoto para la reducción de las emisiones de Gases de Efecto Invernadero (G.E.I.). En 1997 se firmó en Kyoto (Japón) un acuerdo entre los países más industrializados para detener las emisiones de G.E.I., que son el dióxido de carbono y otros cinco gases menos abundantes. Estos gases provocan el aumento de la temperatura media del planeta, conocido como Calentamiento Global, siendo el motivo más importante la combustión de hidrocarburos: $\mathrm{C}_{n} \mathrm{H}_{m}+\mathrm{O}_{2} \rightarrow \mathrm{CO}_{2}+\mathrm{H}_{2} \mathrm{O}+$ Calor

El Protocolo tiene como objetivo reducir las emisiones de los países más industrializados (EEUU, Rusia, Australia, Unión Europea...) en el período 2008-2012 a los niveles de emisión de $\mathrm{CO}_{2}$ en 1990 y a los de 1995 para el resto de G.E.I. Entre los países iberoamericanos sólo España y Portugal deben reducir las emisiones, aunque el resto de países son también firmantes y beneficiarios, ya que son posibles objetivos de inversiones económicas a través de los Mecanismos de Desarrollo Limpio (M.D.L.) [6], [7]. Un M.D.L es una herramienta aprobada por el Protocolo por el que un país con límites de emisiones (como son España y Portugal) justifiquen un exceso de G.E.I. mediante inversiones económicas que reduzcan las emisiones en terceros países; los países lationamericanos son potenciales receptores de estas inversiones por lo que sus políticas energéticas no pueden fomentar las emisiones de G.E.I. En este momento el Protocolo obliga a que España y Portugal reduzcan las emisiones de $\mathrm{CO}_{2}$, pero está pendiente la definición del mismo a partir de 2012, y en este nuevo contexto es de esperar que se les obligue a los países beneficiarios de los M.D.L. a controlar las emisiones si quieren ser receptores de inversiones, por lo que las nuevas instalaciones energéticas deberán emitir menos $\mathrm{CO}_{2}$ que con los combustibles tradicionales. Estas fuentes no emisoras son las renovables (hidráulica, minihidráulica, eólica, solar, biomasa...) o nuclear; debemos citar que la biomasa sí emite $\mathrm{CO}_{2}$ aunque de cara al Protocolo estas emisiones no computan porque se considera que es un almacén de carbono ya producido previamente por otras combustiones como gasóleo, carbón o gas natural. 
El tercer aspecto a considerar es el aumento del consumo de energía a lo largo de las últimas décadas. Desde los años ochenta los países iberoamericanos han aprobado herramientas para reducir el consumo de energía pero el éxito ha sido escaso; el acceso generalizado de la población a las redes eléctricas, el aumento de bienestar y sectores productivos poco eficientes han provocado un aumento en el consumo de forma continua porque en momentos de expansión económica el consumo es creciente mientras que en recesión el consumo se congela, aunque sin llegar a disminuir. En la Tabla 1 y en la Figura 1 podemos ver la evolución del consumo de energía entre 1990 y 2010 para los ocho países iberoamericanos más significativos; en estos países el consumo ha sido creciente en el período 1990-2010; en la línea de la relación entre consumo y crecimiento económico citamos que los países con el mayor aumento de consumo son Chile y Brasil, que también son los que han experimentado la mayor expansión económica.

Tabla 1. Consumo de energía primaria en algunos países iberoamericanos en 1990-2010 (Mtep)

\begin{tabular}{|l|r|r|r|r|r|r|}
\hline & 1990 & 1995 & 2000 & 2005 & 2010 & Aumento 1990-2010 \\
\hline Argentina & 43,3 & 52,4 & 58,9 & 66,7 & 77,1 & $78 \%$ \\
\hline Brasil & 124,5 & 149,3 & 182,8 & 199,2 & 253,9 & $104 \%$ \\
\hline Chile & 12,6 & 17,7 & 22,8 & 27,5 & 28,4 & $125 \%$ \\
\hline Colombia & 22,9 & 26,4 & 25,4 & 27,4 & 32,2 & $41 \%$ \\
\hline España & 91 & 100,5 & 129,2 & 146,5 & 149,7 & $65 \%$ \\
\hline México & 101,6 & 111,7 & 135,8 & 148,2 & 169,1 & $66 \%$ \\
\hline Portugal & 16 & 18,5 & 24,8 & 24,8 & 27,1 & $69 \%$ \\
\hline Venezuela & 46,7 & 54 & 61,9 & 68,3 & 80,3 & $72 \%$ \\
\hline
\end{tabular}

Fuente. B.P. Statistical Review of World Energy

Figura 1: Evolución del consumo de energía en diversos países iberoamericanos

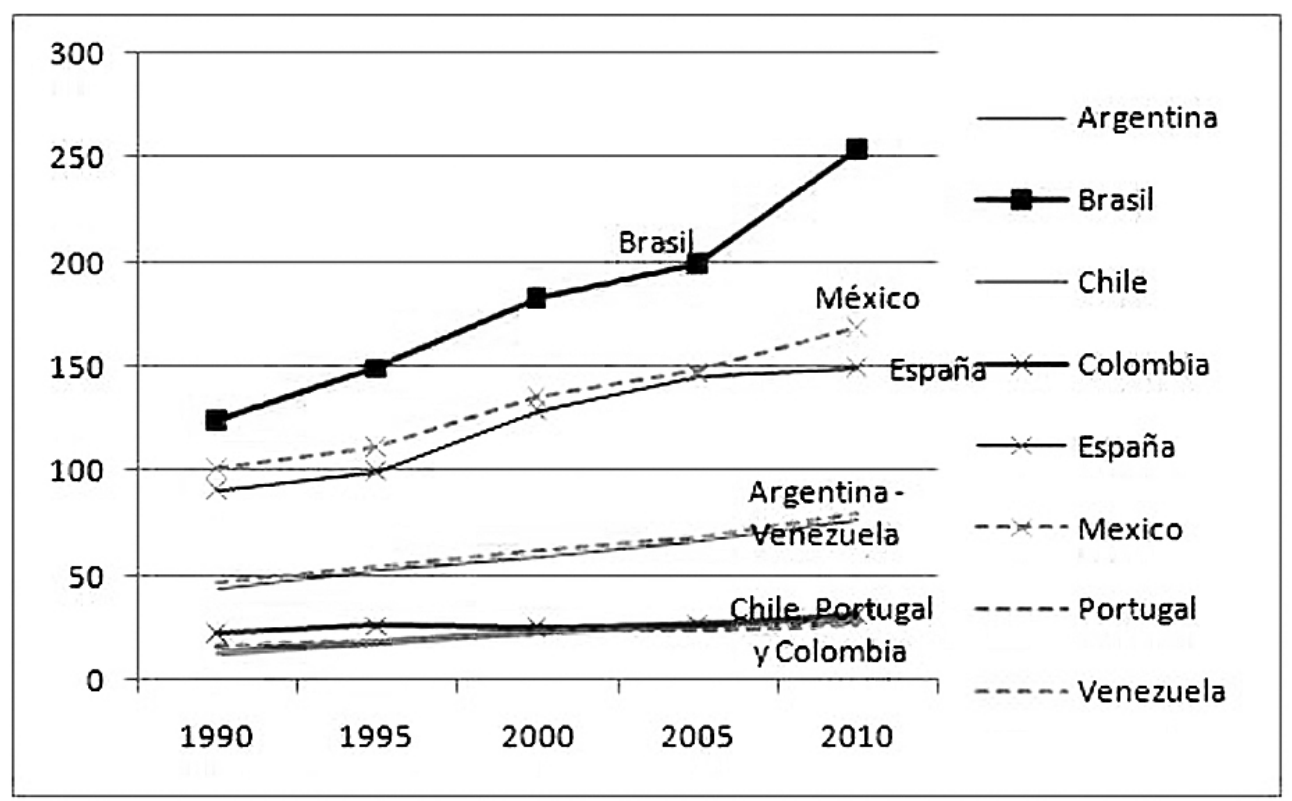


Otros parámetros importantes que debemos considerar en nuestro análisis es el consumo por habitante, que nos puede indicar a grandes rasgos el grado de acceso a la energía del país. En las Figura 2 podemos ver algunos parámetros de consumo de energía por persona entre diversos países iberoamericanos; los países con mayor consumo son España, Venezuela y Portugal, mientras que el país con menor consumo es Colombia; en general el consumo de energía evoluciona de forma paralela al PIB per cápita, cuanto más rico es un país mayor será en términos generales el consumo de energía; en los países que estamos analizando esta relación se cumple exactamente aunque existe una excepción en Venezuela (ver la Figura 3); Venezuela tiene un consumo muy por encima de lo que le correspondería por PIB, según el PIB el consumo de energía de Venezuela debería estar en torno a 1,75 tep/persona cuando el consumo real es casi de 3 tep/persona, de lo que concluimos que existe una mala gestión de la energía.

Tabla 2. Parámetros del consumo de energía en diversos países iberoamericanos

\begin{tabular}{|l|l|l|l|l|l|l|l|l|}
\hline & $\begin{array}{l}\text { Consumo } \\
2010 \\
(\mathrm{Mtep})\end{array}$ & $\begin{array}{l}\text { Población } \\
2010 \\
(\mathrm{millones})\end{array}$ & $\begin{array}{l}\text { Consumo/ } \\
\text { habitante } \\
2010(\text { tep/ } / \\
\text { persona) }\end{array}$ & $\begin{array}{l}\text { PIB nominal } \\
2010(\text { millo- } \\
\text { nes de } €)\end{array}$ & $\begin{array}{l}\text { PIB per } \\
\text { cápita } \\
2010(€\end{array}$ & $\begin{array}{l}\text { Intensidad } \\
\text { energética } \\
\text { (tep/miles } \\
\text { de } €)\end{array}$ & $\begin{array}{l}\text { Reservas } \\
\text { petróleo } \\
\text { (miles millo- } \\
\text { nes tons) }\end{array}$ & $\begin{array}{l}\text { Producción } \\
\text { (miles de } \\
\text { barriles día) }\end{array}$ \\
\hline Argentina & 76,7 & 41,7 & 1,84 & 0,632 & 15156 & 121,36 & 0,3 & 651 \\
\hline Brasil & 253,9 & 203 & 1,25 & 2,182 & 10749 & 116,36 & 2 & 2137 \\
\hline Chile & 28,3 & 16,9 & 1,67 & 0,257 & 15207 & 110,12 & 0 & 0 \\
\hline Colombia & 32,3 & 44,7 & 0,72 & 0,43 & 9620 & 75,12 & 0,3 & 801 \\
\hline España & 149,7 & 46,7 & 3,21 & 1,364 & 29208 & 109,75 & 0 & 0 \\
\hline México & 169,1 & 113,72 & 1,49 & 1,549 & 13621 & 109,17 & 1,6 & 2958 \\
\hline Portugal & 27,1 & 10,76 & 2,52 & 0,246 & 22862 & 110,16 & 0 & 0 \\
\hline Venezuela & 80,2 & 27,63 & 2,90 & 0,367 & 13283 & 218,53 & 30,4 & 2471 \\
\hline Media & 102,2 & 63,1 & 2,0 & 0,9 & 16213,2 & 116,31 & & \\
\hline
\end{tabular}

Fuentes. International Data Base (I.D.B.) de la Oficina del Censo de EEUU, Fondo Monetario Internacional y B.P. Statistical Review 2011

Figura 2: Consumo de energía en 2010 (tep/habitante)

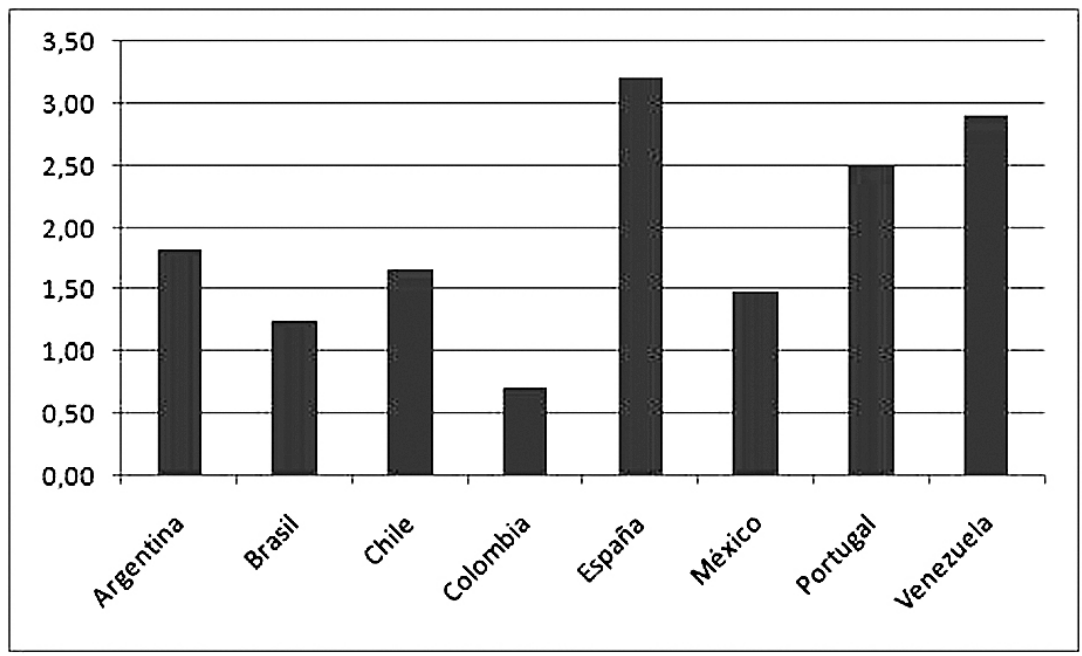

Fuente: B.P. Statistical Review 2011 e International Data Base (I.D.B.) de la Oficina del Censo de EEUU 
Figura 3: Relación entre PIB per cápita (\$) y consumo de energía por persona (tep/persona)

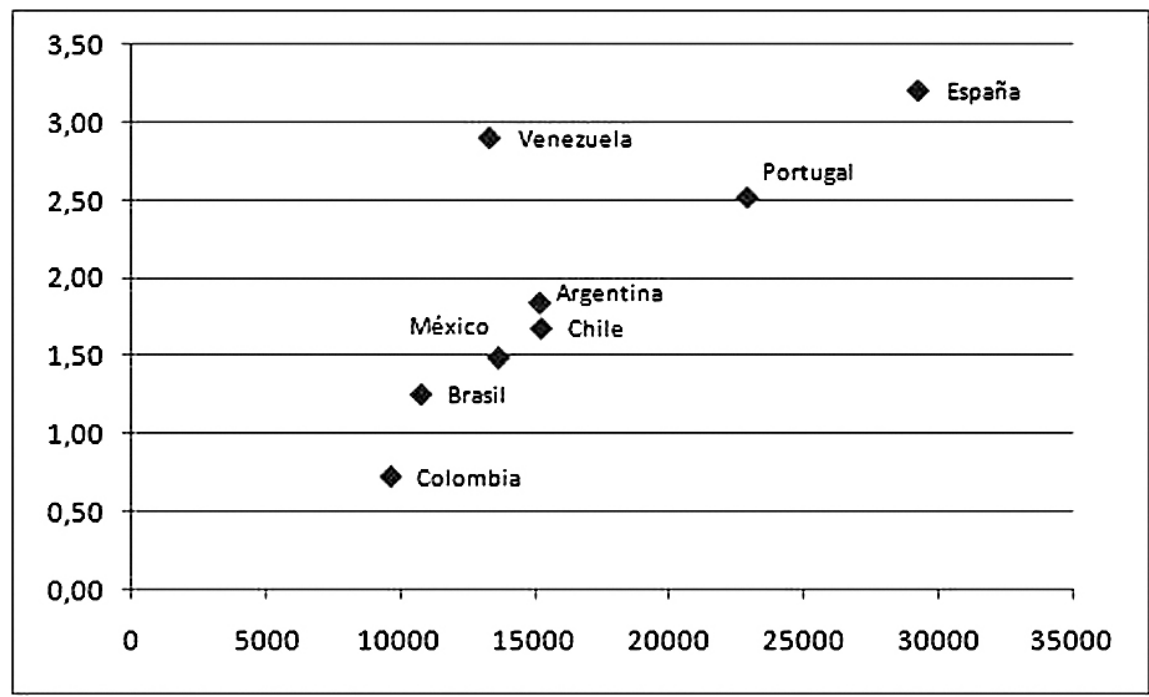

Fuente: International Data Base (I.D.B.) de la Oficina del Censo de EEUU, Fondo Monetario Internacional y B.P. Statistical Review 2011

\section{LA ESTRUCTURA ENERGÉTICA EN LOS PAÍSES IBEROAMERICANOS}

\subsection{TENDENCIAS GENERALES A LO LARGO DE LOS ÚLTIMOS AÑOS}

En función de los tres aspectos analizados (dependencia del petróleo y recursos propios, emisiones de $\mathrm{CO}_{2}$ y aumento del consumo de energía) se definen las políticas energéticas de los países iberoamericanos, adaptándose a la situación particular de cada país. Citamos a continuación las características más importantes en cuanto a las fuentes que utilizan:

I. El petróleo es la primera fuente de energía en todos los países, con la única excepción de Argentina. La dependencia del petróleo un problema grave para Chile y España, ya que en estos países esta fuente supera el $50 \%$ de la energía primaria y carecen de recursos propios; en México también se supera este 50\% aunque la dependencia no es tan problemática porque tiene reservas del hidrocarburo.

II. Colombia y Argentina son los países que han conseguido menos dependencia del petróleo, estando en torno al 30\% de la energía primaria. Ambos países tienen pocos recursos en esta fuente.

III. En Argentina destacamos la importancia del gas natural como fuente más importante (53\% de la energía primaria), siendo el único país que tiene esta característica. En el resto de países el porcentaje del gas natural está por debajo del $40 \%$.

IV. Venezuela se ha centrado en las fuentes de las que dispone recursos (petróleo, gas natural e hidroeléctrica) pero no ha buscado la diversificación. De hecho consideramos que es el país que tiene la estructura energética más débil, tanto por su 
elevado consumo por persona como por la falta de recursos alternativos (Carbón, nuclear o renovables).

V. El carbón es una fuente de energía sólo importante en Portugal o Chile aunque todos los países analizados excepto Venezuela, mantienen un porcentaje mínimo.

VI. Las fuentes de energía renovables tienen únicamente una presencia importante en España y Portugal; esto se explica básicamente en que es una tecnología más cara y de momento sólo está presente en países con elevado P.I.B. per cápita mientras que en el resto la penetración es más lenta.

VII. En la principal característica de la estructura energética de Brasil es la presencia de la gran hidráulica, que supera el 35\% de la energía primaria

Tabla 3. Consumo de energía primaria en diversos países iberoamericanos, medida en millones de toneladas equivalentes de petróleo (2010)

\begin{tabular}{|c|c|c|c|c|c|c|c|}
\hline & Petróleo & Gas Natural & Carbón & Nuclear & Gran Hidráulica & Renovables & Total 2010 \\
\hline Argentina & 25,7 & 39 & 1,2 & 1,6 & 9,2 & 0,4 & 76,7 \\
\hline Brasil & 116,9 & 23,8 & 12,4 & 3,3 & 89,6 & 7,9 & 253,9 \\
\hline Chile & 14,7 & 4,2 & 3,7 & 0 & 4,9 & 0,8 & 28,3 \\
\hline Colombia & 11 & 8,2 & 3,8 & 0 & 9,1 & 0,2 & 32,3 \\
\hline España & 74,5 & 31 & 8,3 & 13,9 & 9,6 & 12,4 & 149,7 \\
\hline México & 87,4 & 62 & 8,4 & 1,3 & 8,3 & 1,7 & 169,1 \\
\hline Portugal & 12,6 & 4,5 & 3,4 & 0 & 3,8 & 2,8 & 27,1 \\
\hline Venezuela & 35,2 & 27,6 & 0 & 0 & 17,4 & 0 & 80,2 \\
\hline
\end{tabular}

Fuente. Fuente:B.P. Statistical Review of Energy de 2011

Tabla 4. Porcentaje de participación de las diversas fuentes de energía primaria en diversos países iberoamericanos

\begin{tabular}{|l|l|l|l|l|l|l|l|}
\hline & Petróleo & Gas Natural & Carbón & Nuclear & Gran hidráulica & Renovables & Total \\
\hline Argentina & $34 \%$ & $51 \%$ & $2 \%$ & $2 \%$ & $12 \%$ & $1 \%$ & $100 \%$ \\
\hline Brasil & $46 \%$ & $9 \%$ & $5 \%$ & $1 \%$ & $35 \%$ & $3 \%$ & $100 \%$ \\
\hline Chile & $52 \%$ & $15 \%$ & $13 \%$ & $0 \%$ & $17 \%$ & $3 \%$ & $100 \%$ \\
\hline Colombia & $34 \%$ & $25 \%$ & $12 \%$ & $0 \%$ & $28 \%$ & $1 \%$ & $100 \%$ \\
\hline España & $50 \%$ & $21 \%$ & $6 \%$ & $9 \%$ & $6 \%$ & $8 \%$ & $100 \%$ \\
\hline México & $52 \%$ & $37 \%$ & $5 \%$ & $1 \%$ & $5 \%$ & $1 \%$ & $100 \%$ \\
\hline Portugal & $46 \%$ & $17 \%$ & $13 \%$ & $0 \%$ & $14 \%$ & $10 \%$ & $100 \%$ \\
\hline Venezuela & $44 \%$ & $34 \%$ & $0 \%$ & $0 \%$ & $22 \%$ & $0 \%$ & $100 \%$ \\
\hline Media & $45 \%$ & $26 \%$ & $7 \%$ & $2 \%$ & $17 \%$ & $3 \%$ & $100 \%$ \\
\hline
\end{tabular}

Fuente. Elaboración propia a partir de datos del B.P. Statistical Review de 2011

A continuación haremos el análisis de las distintas fuentes de energía por países. La diversificación energética es la solución al creciente aumento de la demanda de energía por y los efectos negativos de la subida de los precios del petróleo y [8]. El aumento en el consumo de energía de las últimas décadas ha originado problemas de abastecimiento, es difícil encontrar fuentes alternativas a las convencionales (petróleo, gas natural o carbón) con bajas emisiones de dióxido de carbono a un precio razonable; la mejor solución sería un nuevo modelo energético basado en las fuentes renovables, la reducción del consumo y el fomento de la eficiencia ener- 
gética [9], [10] pero las herramientas desarrolladas para conseguir dicho modelo han fracasado por lo que las nuevas tendencias se dirigen hacia la diversificación entre las fuentes. El primer parámetro que condiciona esta diversificación es el precio de la producción de cada $\mathrm{kWh}$ útil; esto es muy difícil de abordar con un parámetro absoluto, ya que no es lo mismo hacerlo para transporte o producción de energía eléctrica que para consumo térmico aunque a nivel orientativo vamos a centrarnos en la producción eléctrica; para producir un kWh de energía eléctrica de origen nuclear necesitamos unos $5 \mathrm{c} €$, para producir un $\mathrm{kWh}$ usando gas natural necesitamos aproximadamente unos $8 \mathrm{c} €$ mientras que si utilizamos carbón o gran hidráulica el coste es de unos 12 céntimos. En el caso de utilizar fuentes renovables como la eólica o la solar los precios de generación se disparan hasta los 16 c€ ó $40 \mathrm{c} €$; en la Figura 4 podemos ver los precios estimados de producción. No se incluye la producción de electricidad usando productos petrolíferos porque son muy escasos y se trata de una tecnología no habitual para la producción de electricidad aunque está en orden del carbón o gran hidráulica.

Figura 4: Precio estimado de producción del $\mathrm{kWh}^{1}$

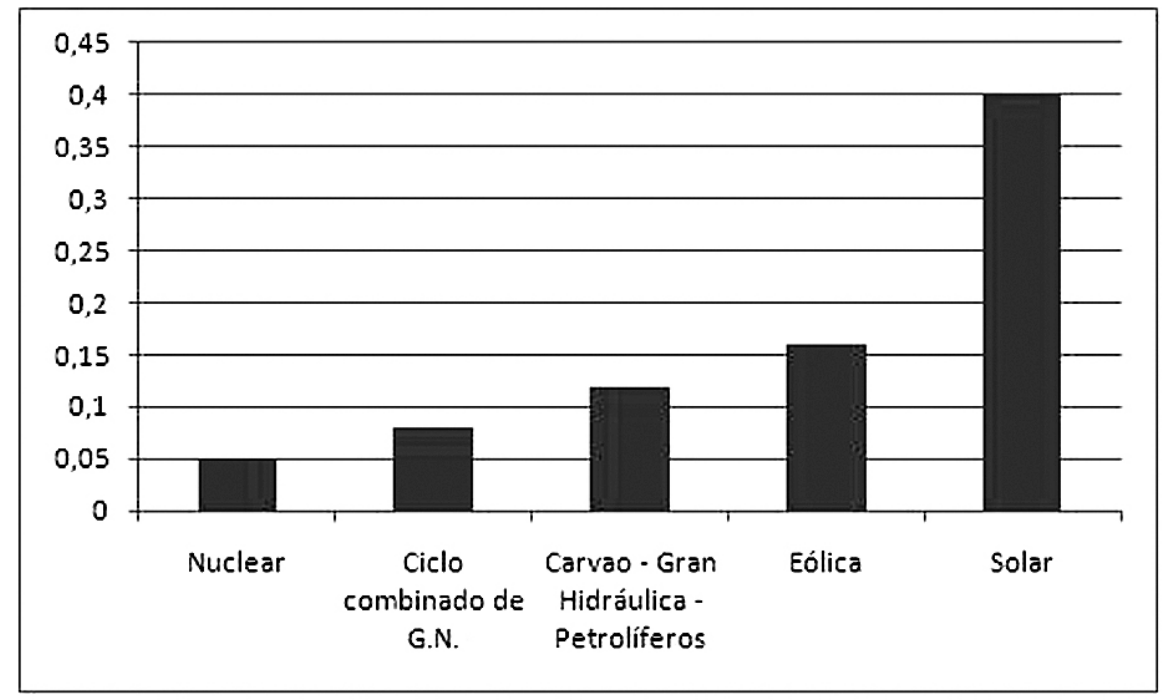

Otra característica importante a la hora de elegir una fuente es la disponibilidad anual; en el caso de la producción eléctrica las centrales térmicas (nucleares, gas natural, carbón...) permiten un funcionamiento durante un número de horas máximo a lo largo del año, unas 8.000 horas, ya que sólo deben estar apagadas por motivos

\footnotetext{
${ }^{1}$ Estos valores son orientativos, y deben considerarse con las correspondientes particularidades ya que los costes no son los mismos en todos los países y hay otros factores que también intervienen como el tamaño de las centrales
} 
de mantenimiento o fallos puntuales. Este funcionamiento es muy superior a las centrales que utilizan las fuentes renovables como la eólica (entre 2.500 y 3.000 horas equivalentes) o la solar (en torno a 1.500 a 2.000 horas nominales). Los combustibles convencionales están disponibles siempre que los necesitamos (podemos disponer de ellos según la demanda) mientras que las fuentes renovables no pueden regularse en función de la demanda, ya que están condicionadas a la meteorología.

\subsection{PETRÓLEO Y GAS NATURAL}

Son las dos fuentes más importantes y a su vez más relacionadas entre sí. El petróleo es la fuente de energía más consumida en el mundo y en los países analizados supera el 50\% de la energía primaria en España, México y Chile. El gas natural es una fuente alternativa al petróleo en sus usos (doméstico, generación de calor en calderas, producción eléctrica...) aunque las reservas de ambos hidrocarburos se concentran en general en los mismos países; el precio por kWh de gas natural es algo más bajo aunque tampoco se puede considerar un combustible realmente alternativo al petróleo porque una subida en el precio de este combustible suele reflejarse inmediatamente en un encarecimiento del gas natural, así un aumento de la presencia del gas natural mejora la diversificación pero no consigue blindar la economía ante nuevas subidas de precio. Una ventaja importante del gas natural es el alto rendimiento de los ciclos combinados a la hora de producir electricidad y un impacto ambiental menor que en la combustión de petróleo o carbón.

\subsection{CARBÓN}

El carbón es un combustible utilizado en general para la generación de energía eléctrica. Sus reservas son muy importantes, superiores en cientos de años al petróleo o gas natural, pero tiene como desventaja el precio y la alta emisión de Gases de Efecto Invernadero por cada $\mathrm{kWh}$ producido (a la hora de producir electricidad el rendimiento de un ciclo de vapor que quema carbón es más bajo que en el ciclo combinado que consume gas natural). El carbón está presente en muchos países y no podemos considerar que sea un combustible escaso, pero durante las últimas décadas se explotado los yacimientos más accesibles y los recursos disponibles ya no son fácilmente extraíbles; no obstante los países mantienen el consumo de carbón aunque sea a mayor precio para favorecer la diversificación, con la excepción de Venezuela.

\subsection{ENERGÍA TERMONUCLEAR}

La energía nuclear es la que mejor representa la diversificación de las fuentes aunque sólo existen plantas en cuatro países (México, Argentina, Brasil y España). El que tiene mayor potencia implantada es España (seis centrales con potencia total de 7.730 MW) y la nuclear es origen del $9 \%$ de la energía primaria y un 19\% de la producción eléctrica; en el resto de países esta fuente es testimonial (potencia entre 1.000 MW y 2.000 MW y menos del 3\% de la energía primaria consumida en el país. Además de las centrales nucleares existentes algunos países estudian incorporarse a este grupo 
(Venezuela ${ }^{2}$, Chile ${ }^{3}$ y Colombia ${ }^{4}$ se han planteado abrir nuevas plantas incluso con estudios en profundidad [11]) mientras que otros se plantean aumentar la potencia existente; por el contrario el accidente nuclear de Fukushima ha resucitado el movimiento antinuclear y desatando nuevas protestas para que se cierren las centrales que están ya funcionando. Los argumentos sobre el debate son muy interesantes, y válidos en los dos sentidos. Las razones a favor de esta fuente de energía son básicamente el precio, disponibilidad, abundancia de combustible (es un recurso abundante en muchos países) y que no emite G.E.I.; existe una tabla de impacto ambienta publicada por el Departamento de Energía de los EEUU [12] que valora las distintas fuentes de energía para la producción de electricidad, y concluye que la contaminación producida por la energía nuclear (12.3 ecopuntos) es mucho menor que la producida por el carbón (1066,1 ecopuntos) y gas natural (825,8 ecopuntos) y que la energía nuclear tiene una contaminación del orden de las fuentes renovables; esta clasificación tiene una trampa porque no considera los riesgos de explosiones o accidentes nucleares, que aunque han sido muy pocos las consecuencias fueron terribles.

Nos quedan por analizar los inconvenientes de esta fuente de energía, que han sido abordados por muchos autores, [13], [14], [15] El primero y más importante es que se trata de una tecnología intrínsecamente peligrosa y potencialmente agresiva con el medio ambiente; a diferencia de otras fuentes de energía convencionales (gas natural, carbón o uso de derivados petrolíferos) la energía nuclear cuenta con imponderables que no se pueden controlar, aunque los protocolos de actuación son muy estrictos hay riesgos que pueden dar lugar a grandes problemas; el ejemplo más claro es el accidente de Fukushima, con protocolos que se cumplieron a la perfección, pero con un terremoto diez veces mayor a los registrados. Las consecuencias sobre las personas son muy nocivas incluyendo la generación de leucemia, cánceres de todo tipo, alteraciones genéticas, esterilidad...; sobre el medio ambiente las consecuencias también son muy graves siendo afectados durante miles de años tanto el agua como los suelos; en el caso de la Central Nuclear de Chernobyl los terrenos en un radio de unos $20 \mathrm{kms}$ son irrecuperables y no está permitido ni acercarse por los efectos nocivos de la radiación acumulada. El otro gran problema de esta fuente es la gestión y almacenamiento de los residuos; los elementos químicos radiactivos tienen una vida mucho más allá del tiempo de uso (se calcula que la radiactividad podría durar miles de años) y los únicos tratamientos actuales son la reducción de volumen y un almacenamiento en condiciones seguras, pero no se ha conseguido inertizar la actividad radiactiva.

Estos dos hechos son aceptados como inconvenientes de forma unánime, y a esto se debe añadir la rentabilidad a largo plazo; los costes de producción son menores cuando usamos de otras fuentes cuando se excluye el deterioro ambiental; los grupos

\footnotetext{
$2 \mathrm{http}: / /$ www.jornada.unam.mx/2008/09/29/index.php?section=mundo\&article=035n $1 \mathrm{mun}$

3 http://www.lanacion.cl/noticias/site/artic/20110317/pags/20110317203038.html

${ }^{4} \mathrm{http} / / /$ www.eltiempo.com/blogs/capitolium_mundial/2009/09/colombia-con-derecho-a-la-ener-1.php
} 
ecologistas afirman que el coste real de producir un $\mathrm{kWh}$ de origen nuclear es mucho mayor porque debería incluirse el impacto ambiental, con unos residuos peligrosos y cuya gestión tiene unos costes desconocidos, ya que se debe costear su custodia durante los años que durará el la desintegración total. Debemos citar un último inconveniente; las centrales nucleares tardan del orden de unos diez año en ponerse en funcionamiento desde que se proyectan, a diferencia de las centrales eólicas (dos años) y fotovoltaicas (un año); con un plazo tan largo podemos encontrarnos que al entrar en funcionamiento las necesidades para las que se crean hayan cambiado y la nueva central no tendrían objeto.

\subsection{GRAN HIDRÁULICA Y FUENTES RENOVABLES}

Analizamos estas dos fuentes de energía en conjunto, debido a que utilizan recursos renovables. La gran hidráulica son aquellas centrales de producción hidroeléctricas de potencia mayor a $50 \mathrm{MW}$, y se consideran aparte al resto de renovables (hidráulica convencional, eólica, solar fotovoltaica, solar termoeléctrica...) porque la obra civil necesaria para estas centrales tiene un impacto irreparable, por lo que no recibe las ayudas del resto de renovables. La gran hidráulica consigue un precio por $\mathrm{kWh}$ bajo por economías de escalas pero en general las fuentes renovables suponen un coste mucho mayor por lo que deben recibir subvenciones; este sobrecoste unido a la falta de disponibilidad (dependen de la meteorología) son los mayores inconvenientes que presentan mientras que su principal ventaja es que el impacto ambiental es mucho menor y no provocan emisiones de G.E.I.

\section{CONCLUSIONES: MODELOS ENERGÉTICOS EN LOS PAÍSES IBERO- AMERICANOS}

En el presente artículo hemos estudiado los ocho países más significativos de Iberoamérica, y vemos que cada uno de los ocho tiene una casuística particular, que impide que se analicen en su conjunto.

El país que tiene una mayor debilidad es Venezuela; su estructura está basada en petróleo, gas natural e hidroeléctrica, que son los recursos nacionales. Hasta ahora no existe una política de diversificación, no se da la entrada a las fuentes alternativas (renovables, nuclear..) ni se utiliza con este fin el carbón.

En el resto de países la situación es muy variada. España ha hecho importantes esfuerzos para facilitar la entrada de nuevas fuentes (es el único que asegura al menos un 5\% de cada fuente) pero tiene su gran debilidad en la dependencia de petróleo; Portugal tiene mejor diversificación aunque carece de centrales nucleares y dependencia del petróleo; México y Brasil tienen una estructura adaptada a los recursos nacionales (petróleo o gas natural) aunque carece de fuentes renovables y energía nuclear mientras que Chile y Brasil están en una situación intermedia a los anteriores fomentando la gran hidroeléctrica. Colombia se caracteriza por un consumo diversificado pero tiene su gran fortaleza en un consumo moderado y una estructura 
eficiente. Argentina tiene una alta diversificación, destacándose la presencia del gas natural pero la presencia de fuentes renovables es muy escasa.

\section{REFERENCIAS BIBLIOGRÁFICAS}

[1] MIJARES, VÍCTOR M.: "Petróleo y conflicto en la estructura internacional: aproximación a los fundamentos básicos del conflicto internacional generado por el control de las principales fuentes mundiales de petróleo", Revista Venezolana de Análisis de Coyuntura, vol. I - No2, 43-57

[2] OCAMPO TELLEZ, EDGAR. "Encarecimiento del petróleo: preludio del agotamiento". Elementos: Ciencia y Cultura, $\mathrm{n}^{\circ} 63$ (2006), 53-59

[3] KETELSEN, UWE; RUBINSOHN: "El petróleo y la economía global". Economía exterior: estudios de la revista Política Exterior sobre la internacionalización de la economía española, $\mathrm{n}^{\circ}$ 37, 2006, 103-110

[4] BAZÁN PERKINS, SERGIO. "La energía nuclear, una alternativa de sustentabilidad para resolver la demanda eléctrica en México". Ingeniería.Investigación y Tecnología, no 3 (2005) 187-205.

[5] CÓRDOVA SÁEZ, KARENIA. "Criterios para la definición de ámbitos territoriales prioritarios en la implantación de fuentes alternas de energía en Venezuela". Terra Nueva Etapa, n029 (2004) 35-53.

[6] SANHUEZA, EUGENIO. "Sucio aprovechamiento del M.D.L. Hay que cambiar el Protocolo de Kyoto". Interciencia, vol. 35, nº 11 (2010) págs.. 797-799.

[7] LINKOHR, ROLF. "La política energética lationamericana: entre el Estado y el mercado". Nueva Sociedad, no 204 (2006) 90-103

[8] CASTRO DÍAZ-BALART, FIDEL. "Energía nuclear: ¿peligro ambiental o solución para el siglo XXI?. Editorial Grijalbo Mondadori, Barcelona, 1999

[9] MENÉNDEZ PÉREZ, EMILIO. "Eficiencia energética: una necesidad en un entorno complejo". Ingeniería Química, no 413 (2004) 148-154.

[10] SANTAMARTA FLÓREZ, JOSÉ "Las energías renovables son el futuro". Mapping, no99 (2005) 68-83

[11] ROTHWEL, GEOFFREY. “Energía nuclear en Chile?: Los costos y beneficios de la opción de construir una central nuclear en 2020. Estudios públicos, $\mathrm{n}^{\circ} 112$ (2008) 6-30

[12] US Departament of Energy, Council for Renewable Energy Education. Tabla de Ecopuntos según las fuentes de energía, toneladas por GWh producido.

[13] BRAVO VILLA, CARLOS. "Energía nuclear: oscura, peligrosa e insostenible". Economía Industrial, no 369 (2008) 161-168.

[14] SANTAMARTA FLÓREZ, JOSÉ. "El cierre de Chernóbil no acaba con la pesadilla nuclear". Papeles del Este: Transiciones poscomunistas, $\mathrm{n}^{\circ} 2$ (2011)

[15] MOBERG, ASA; BIGAS, JORDI; MORÓN, JAUME. "La energía nuclear en crisis: antes y después de Chernobil". Lerna, Barcelona, 1987. 